\title{
Comparison of CFD simulations and experiments for stratified air-water flows in pipes
}

\author{
Gabriele Chinello, first author ${ }^{1}$ \\ Department of Engineering, Glasgow Caledonian University, United Kingdom \\ Glasgow Caledonian University, Cowcaddens road, G40BA Glasgow, United Kingdom \\ gabriele.chinello@gcu.ac.uk
}

Anis Awal Ayati, second author

Department of Mathematics, University of Oslo, Norway

University of Oslo, Department of Mathematics, N-0316 Oslo, Norway

awalaa@math.uio.no

Don McGlinchey, third author

Department of Engineering, Glasgow Caledonian University, United Kingdom

Glasgow Caledonian University, Cowcaddens road, G40BA Glasgow, United Kingdom

D.McGlinchey@gcu.ac.uk

Gijs Ooms, fourth author

Laboratory for Aero and Hydrodynamics, Process and Energy Department, Delft University of Technology, The Netherlands

Delft University of Technology, Laboratory for Aero and Hydrodynamics, Leeghwaterstraat 21, 2628 CA Delft, The Netherlands

G.Ooms@tudelft.nl

\section{Ruud Henkes, fifth author}

Laboratory for Aero and Hydrodynamics, Process and Energy Department, Delft University of Technology, The Netherlands

Shell Projects \& Technology, The Netherlands

Delft University of Technology, Laboratory for Aero and Hydrodynamics, Leeghwaterstraat 21, 2628 CA Delft, The Netherlands

\section{R.A.W.M.Henkes@tudelft.nl}

\footnotetext{
${ }^{1}$ Corresponding author.
} 


\section{ABSTRACT}

Stratified gas-liquid flow is a flow regime typically encountered in multiphase pipelines. The understanding and modelling of this regime is of engineering importance especially for the oil and gas industry. In this work simulations have been conducted for stratified air-water flow in pipes with the Computational Fluid Dynamics package Fluent 17.1. The simulation results were compared with some of the latest experimental results found in the literature. A comparison between the simulated velocity and kinetic energy profiles and the experimental results obtained with the Particle Image Velocimetry technique was conducted. The characteristics of the interfacial waves were also extracted and compared with the experiments. It is shown that proper damping of the turbulence close to the interface is needed to obtain agreement with the experimental pressure drop and liquid hold-up.

\section{INTRODUCTION}

Stratified two-phase flow is a flow regime typically encountered in gas transportation pipelines, where small amounts of hydrocarbon condensate or water are present, and in multiphase oil/gas pipelines. For the pipeline design, which includes the proper integrity and the optimal production rates, it is important to obtain accurate predictions of the pressure gradient and of the liquid hold-up (i.e. the liquid accumulation).

Early studies have focused on the determination of semi-empirical correlations to obtain the pressure gradient and hold-up, e.g. Lockhart and Martinelli [1] and Chisolm [2]. The one-dimensional mechanistic approach, as initially developed by Taitel and Dukler [3], is still widely used for engineering pipeline design; that method applies a local momentum balance equation for each phase after averaging over the pipe cross-sectional area. Due to the averaging process, the information in the direction normal to the main flow is lost. Hence in 1-D models the main issues are related to the formulation of closure relations for the interfacial stresses and for the 
wall shear stresses. Many studies have focused on the derivation of empirical closure relations, see Espedal [4] for a detailed review. The focus in more recent studies is on how detailed turbulence modelling can help to develop closure relations for the 1-D models, see e.g. Biberg [5].

One-dimensional models are widely used in the oil and gas industry and they are employed in commercial software tools (such as OLGA and LedaFlow) to simulate pipeline systems. Nowadays, this still is the only practical solution for simulating long pipelines due to the extremely high computational costs associated with multidimensional CFD simulations. However, 1-D models have limitations when 3-D effects are relevant and a detailed flow field computation is necessary. Inaccuracies have been observed in the simulations of complex geometries and for low flow in upward inclined pipes, [6-8]. In these cases CFD becomes an interesting approach since it can help to improve the existing 1-D models. Furthermore, CFD is receiving increasing attention due to the development of software that directly combines CFD and 1-D simulations. This is quite attractive, since a detailed flow field computation will be applied only where needed, thus maintaining a reasonable computational time.

So far various studies have been conducted to simulate stratified two-phase flow in channels and pipelines, using the Reynolds-Averaged Navier-Stokes (RANS) equations. Among these studies, two main categories may be identified: "one-fluid" formulation studies, in which only one set of equations is solved for both phases, and "two-fluid" formulation studies, where the equations are solved for each of the phases [9]. The present study is focussed on the one-fluid method, as this is most 
suitable for separated flows (like stratified flow), while the two-fluid approach is more suitable for dispersed flow (i.e. liquid droplets carried by a gas flow, or gas bubbles carried by a liquid flow). The considered flow velocities are so low that there is stratified flow, with turbulence and with interfacial waves, but without the formation and entrainment of bubbles or droplets.

Within the one-fluid studies, Holmås et al. [10] used the commercial code COMET to simulate stratified two-phase flow in a channel with the volume of fluid (VOF) method. Periodic boundary conditions were employed and a fixed pressure gradient was imposed. The turbulence was solved both with the $k-\varepsilon$ RNG model and with the $k-\omega$ SST model. The predicted velocity and turbulent kinetic energy profiles were compared with the experiments by Akai et al. [11] for a smooth interface and by Lorencez et al. [12] for a wavy interface. Both for smooth and wavy interface the gas and liquid flow rates disagreed with the experimental findings. Therefore Holmås et al. [10] concluded that the turbulence models had to be modified in order to correctly predict the turbulence at the gas-liquid interface. The same conclusion was drawn by Terzuoli et al. [13], who used the commercial codes CFX 10.0 and Fluent 6.1 to simulate the experiment of air-water in a channel by Fabre et al. [14]. The VOF method was employed, with the $k-\omega$ SST model for the turbulence. Lo and Tomasello [15] performed three-dimensional simulations of gas-liquid flow in pipes with the commercial code Star-CD, using the VOF method. Three turbulence models were tested: standard $k-\varepsilon$, standard $k-\omega$, and $k-\omega$ SST. The pressure drop and hold-up were compared with the experimental results of Espedal [4] for air-water flow in a pipe. The pressure drop was overestimated while the hold-up was underestimated by all 
the three turbulence models, when the turbulence viscosity was not artificially reduced close to the interface.

When the source term proposed by Egorov [16] is added to the $\omega$ equation, a considerable improvement in the predictions was obtained by Lo and Tomasello. This term equally damps the turbulence at both sides of the interface similarly to the damping near a solid wall. However, a disadvantage of the source term is that has to be tuned and moreover it has a dependence on the size of the cell employed at the interface. A method is presented by Lo and Tomasello to resolve the mesh size dependence problem, but as reported by the authors it needs further testing and development. The mentioned studies show that the correct simulation of gas-liquid flow with RANS in CFD could not yet be established, and more investigation is needed.

In this work, RANS-based CFD simulations are carried out for gas-liquid pipe flow with Fluent 17.1 using the VOF method, which is a one-fluid approach. The simulation results are compared with the experiments of Espedal [4], Birvalski [1719] and Ayati et al. [20,21]. Two numerical approaches are employed. For the cases of Espedal and Birvalski the entire experimental pipe length is simulated, while for the case of Ayati et al. the computational pipe length is only a portion of the experimental one and periodic boundary conditions are imposed. The last approach is employed to capture the details of the structure of the interfacial waves by adopting a very refined mesh which would not be computationally feasible if the full pipe length was simulated. As Particle Image Velocimetry was used in the experiments by both Birvalski and Ayati et al., the simulation results can be 
compared with the available experimental velocity profiles. In addition, from the experiments of Ayati et al., wave features such as the peak frequency, average wave length, average amplitude, average steepness and average celerity are available, which can also be used for the validation of the simulation results.

To the authors' knowledge this is the first study in the open literature to investigate the performance of CFD with the VOF method, with and without damping at the interface, by comparing simulation results with experimental results for the pressure drop, the liquid hold-up, and the velocity (PIV) as well as for the characteristics of the waves for stratified gas-liquid flow in pipes. The main finding of this investigation is that while bulk quantities such as the pressure drop and hold-up can be predicted well (up to $\pm 10 \%$ ), when turbulence is damped at the gas-liquid interface, the prediction of the velocity profiles and of the wave characteristics is largely unsatisfactory both with and without turbulence damping at the interface. The aim of this paper is to assess the actual capability of CFD with the VOF method against some of the latest high quality experimental results to steer the future development in modelling of gas-liquid stratified flow in pipes.

\section{GOVERNING EQUATIONS}

Three-dimensional simulations are conducted for air-water flow in a pipe with the CFD package Fluent 17.1. The continuity and momentum equations are given by

$$
\begin{gathered}
\frac{\partial \rho}{\partial t}+\nabla \cdot(\rho \vec{v})=0 \\
\frac{\partial}{\partial t}(\rho \vec{v})+\nabla \cdot(\rho \vec{v} \vec{v})=-\nabla p+\nabla \cdot\left(\bar{\tau}+\bar{\tau}_{t}\right)+\rho \vec{g}+\vec{F}
\end{gathered}
$$


where $\vec{F}$ is a force term that can be used to model the effect of surface tension. The molecular stress tensor in (2) is defined as

$$
\bar{\tau}=\mu\left[\left(\nabla \vec{v}+\nabla \vec{v}^{T}\right)-\frac{2}{3} \nabla \cdot \vec{v} I\right]
$$

The turbulent stress tensor (Reynolds stress) in (2) is defined with the Boussinesq eddy-viscosity approximation

$$
\bar{\tau}_{t}=\mu_{t}\left[\left(\nabla \vec{v}+\nabla \vec{v}^{T}\right)-\frac{2}{3}(\nabla \cdot \vec{v}+\rho k) I\right]
$$

in which $I$ is the unit tensor. The Volume of Fluid (VOF) technique is employed to track the interface between the two phases, which thus considers the two phases as one single mixture. The mixture density and viscosity are given by the value of the volume fraction in the cell $(\alpha)$, according to the following expressions

$$
\begin{aligned}
& \rho=\alpha_{2} \rho_{2}+\left(1-\alpha_{2}\right) \rho_{1} \\
& \mu=\alpha_{2} \mu_{2}+\left(1-\alpha_{2}\right) \mu_{1}
\end{aligned}
$$

To obtain the volume fraction in each cell the following transport equation is solved for one of the phases (the second phase is selected)

$$
\frac{\partial \alpha_{2} \rho_{2}}{\partial t}+\nabla \cdot\left(\alpha_{2} \rho_{2} \vec{v}_{2}\right)=0
$$

The volume fraction of the other phase (the first phase) is computed based on the following constraint

$$
\alpha_{1}+\alpha_{2}=1
$$

\section{Turbulence modelling}

The $k-\omega$ shear stress transport (SST) model is used [22]. This model has been chosen because it gives better results than the standard $k-\omega$ model and than the standard $k$ - 
$\varepsilon$ model when simulating air-water flow without any artificial damping at the interface [15]. In the $k$ - $\omega$ SST the turbulence viscosity is given by

$$
\mu_{t}=\frac{\rho k}{\omega} \frac{1}{\max \left[\frac{1}{\alpha^{*}}, \frac{S F_{2}}{a_{1} \omega}\right]}
$$

where $S$ is the strain rate magnitude $S=\sqrt{2 S_{i j} S_{i j}} \quad$ and $S_{i j}=\frac{1}{2}\left(\frac{\partial v_{i}}{\partial x_{j}}+\frac{\partial v_{j}}{\partial x_{i}}\right)$. The transport equations for the turbulent kinetic energy $k$ and for the specific dissipation rate $\omega$ are

$$
\begin{gathered}
\frac{D \rho k}{D t}=\frac{\partial}{\partial x_{j}}\left[\left(\mu+\frac{\mu_{t}}{\sigma_{k}}\right) \frac{\partial k}{\partial x_{j}}\right]+\min \left(\mu_{t} S^{2}, 10 \rho \beta^{*} k \omega\right)-\rho \beta^{*} k \omega \\
\frac{D \rho \omega}{D t}=\frac{\partial}{\partial x_{j}}\left[\left(\mu+\frac{\mu_{t}}{\sigma_{\omega}}\right) \frac{\partial \omega}{\partial x_{j}}\right]+\frac{\alpha}{v_{t}} \min \left(\mu_{t} S^{2}, 10 \rho \beta^{*} k \omega\right)-\rho \beta \omega^{2} \\
+2\left(1-F_{1}\right) \rho \frac{1}{\sigma_{\omega, 2} \omega} \frac{\partial k}{\partial x_{j}} \frac{\partial \omega}{\partial x_{j}}+S_{\omega}
\end{gathered}
$$

The model constants are given in table 1 , and the model coefficients are as follows:

$$
\begin{gathered}
\sigma_{k}=\frac{1}{\frac{F_{1}}{\sigma_{k, 1}}+\frac{1-F_{1}}{\sigma_{k, 2}}} ; \quad \sigma_{\omega}=\frac{1}{\frac{F_{1}}{\sigma_{\omega, 1}}+\frac{1-F_{1}}{\sigma_{\omega, 2}}} ; \quad \alpha^{*}=\alpha_{\infty}^{*}\left(\frac{\alpha_{0}^{*}+R e_{t} / R_{k}}{1+R e_{t} / R_{k}}\right) \\
\beta^{*}=\beta_{i}^{*}=\beta_{\infty}^{*}\left(\frac{4 / 15+\left(R e_{t} / R_{\beta}\right)^{4}}{1+\left(R e_{t} / R_{\beta}\right)^{4}}\right) ; \quad \alpha=\frac{\alpha_{\infty}}{\alpha^{*}}\left(\frac{\alpha_{0}+R e_{t} / R_{\omega}}{1+R e_{t} / R_{\omega}}\right) \\
\beta=\beta_{i}=F_{1} \beta_{i, 1}+\left(1-F_{1}\right) \beta_{i, 2} ; \quad \alpha_{\infty}=F_{1} \alpha_{\infty, 1}+\left(1-F_{1}\right) \alpha_{\infty, 2} \\
\alpha_{\infty, 1}=\frac{\beta_{i, 1}}{\beta_{\infty}^{*}}-\frac{\kappa^{2}}{\sigma_{\omega, 1} \sqrt{\beta_{\infty}^{*}}} ; \quad \alpha_{\infty, 1}=\frac{\beta_{i, 2}}{\beta_{\infty}^{*}}-\frac{\kappa^{2}}{\sigma_{\omega, 2} \sqrt{\beta_{\infty}^{*}}} ; \quad R e_{t}=\frac{\rho k}{\mu \omega}
\end{gathered}
$$


Table 1: Constants in the $k-\omega$ SST turbulence model [23]

\begin{tabular}{|c|c|c|c|c|c|c|}
\hline$\sigma_{k, 1}$ & $\sigma_{k, 2}$ & $\sigma_{\omega, 1}$ & $\sigma_{\omega, 2}$ & $a_{1}$ & $\beta_{i, 1}$ & $\beta_{i, 2}$ \\
\hline 1.176 & 1.0 & 2.0 & 1.168 & 0.31 & 0.075 & 0.0828 \\
\hline
\end{tabular}

\begin{tabular}{|c|c|c|c|c|c|c|}
\hline$\alpha_{\infty}^{*}$ & $\alpha_{0}$ & $\beta_{\infty}^{*}$ & $R_{\beta}$ & $R_{k}$ & $R_{\omega}$ & $\kappa$ \\
\hline 1 & $1 / 9$ & 0.09 & 8 & 6 & 2.95 & 0.41 \\
\hline
\end{tabular}

The blending functions are given by

$$
\begin{gathered}
F_{1}=\tanh \left\{\min \left[\max \left(\frac{\sqrt{k}}{0.09 \omega y}, \frac{500 \mu}{\rho y^{2} \omega}\right), \frac{4 \rho k}{\sigma_{\omega, 2} D_{\omega}^{+} y^{2}}\right]\right\}^{4} \\
F_{2}=\tanh \left[\max \left(\frac{2 \sqrt{k}}{0.09 \omega y}, \frac{500 \mu}{\rho y^{2} \omega}\right)\right]^{2}
\end{gathered}
$$

where $y$ is the distance to the closest wall surface and $D_{\omega}^{+}$is

$$
D_{\omega}^{+}=\max \left[2 \rho \frac{1}{\sigma_{\omega, 2} \omega} \frac{\partial k}{\partial x_{j}} \frac{\partial \omega}{\partial x_{j}}, 10^{-10}\right]
$$

The additional source term in the $\omega$ equation $\left(S_{\omega}\right)$ in equation (11) is usually set to zero. However, in order to damp the spurious amount of turbulence at the gas-liquid interface the source term proposed by Egorov [16] has been employed

$$
S_{\omega}=A \Delta n \beta \rho\left(\frac{B 6 \mu}{\beta \rho(\Delta n)^{2}}\right)^{2}
$$


where $\Delta n$ is the cell height normal to the interface, $\beta=0.075$, and $B$ is a tuneable parameter. The term $A$ is the interface area density and is defined as

$$
A=2.0 \alpha|\nabla \alpha|
$$

in which $\alpha$ is the volume fraction. This term $A$ activates the source term in the proximity of the interface $(|\nabla \alpha|=0$ outside the interface) and determines the magnitude of the source term in accordance with the magnitude of the volume fraction gradient. According to Wilcox [24] $\omega$ tends to $6 \mu /\left(\beta \rho\left(\Delta n^{2}\right)\right)$ for $\mathrm{y}^{+}<2.5$ near a smooth wall. Equation (12) thus tries to mimics the asymptotic behaviour of $\omega$ near a smooth wall, reducing the turbulence viscosity at the gas-liquid interface. Substituting equation (12) into equation (11) and rearranging, the following equation is obtained

$$
\begin{array}{r}
\rho \beta \omega^{2}=-\frac{D \rho \omega}{D t}+\frac{\partial}{\partial x_{j}}\left[\left(\mu+\frac{\mu_{t}}{\sigma_{\omega}}\right) \frac{\partial \omega}{\partial x_{j}}\right]+\frac{\alpha}{v_{t}} \min \left(\mu_{t} S^{2}, 10 \rho \beta^{*} k \omega\right) \\
+2\left(1-F_{1}\right) \rho \frac{1}{\sigma_{\omega, 2} \omega} \frac{\partial k}{\partial x_{j}} \frac{\partial \omega}{\partial x_{j}}+A \Delta n \beta \rho\left(\frac{B 6 \mu}{\beta \rho(\Delta n)^{2}}\right)^{2}
\end{array}
$$

where the left-hand side is the standard $\omega$ destruction term and the right-hand side contains all the other terms plus the newly introduced source term. The implicit assumption performed by Egorov is that the only relevant terms at the gas-liquid interface are the destruction and the source term. Under this assumption the equation above can then be rearranged to

$$
\omega=\sqrt{A \Delta n} B\left(\frac{6 \mu}{\beta \rho(\Delta n)^{2}}\right)
$$


This enforces a large value of $\omega$ at the interface, which is quite similar to what is imposed near a smooth wall.

\section{MESH}

To reduce the computational time only half the pipe was simulated (assuming symmetry with respect to the midplane of the pipe). The characteristics of the different meshes used in this work are given in table 2.

Table 2: Cell distribution of the three different meshes employed

\begin{tabular}{|l|c|c|c|}
\hline Mesh & $\begin{array}{c}\text { No. cells in } \\
\text { pipe cross } \\
\text { section }\end{array}$ & $\begin{array}{c}\text { No. cells in } \\
\text { pipe axial length }\end{array}$ & cells \\
\hline Mesh 1 & 190 & 449 & 85,310 \\
\hline Mesh 2 & 480 & 674 & 323,520 \\
\hline Mesh 3 & 902 & 899 & 810,898 \\
\hline Mesh 4 & 1040 & 600 & 624,000 \\
\hline
\end{tabular}

The meshes 1, 2 and 3 were used to simulate the entire pipe length, which is $10.3 \mathrm{~m}$ for the experiments by Birvalski [17] and $18 \mathrm{~m}$ for the experiments by Espedal [4]. Mesh 4 was used to simulate one meter of pipe for the experiments by Ayati et al. $[20,21]$. The diameter was set to $0.05 \mathrm{~m}, 0.06 \mathrm{~m}$ and $0.1 \mathrm{~m}$ for the configurations used by Birvalski, Espedal and Ayati et al., respectively. Figure 1 shows the cell distribution over the cross section for the three different meshes employed in this study. For each simulation the size of the first cell near the wall was adjusted to assure that it corresponds to a $y^{+}$value between 25 and 80 . This size was determined 
with the log-law by imposing $y^{+}=30$ at the gas side and taking the gas mean velocity from the experimental values. This size was used for all the first cells near the wall.

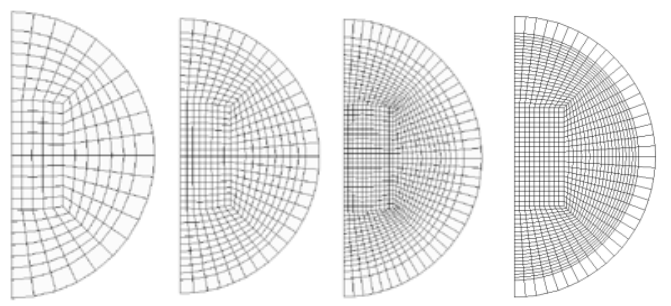

Figure 1: Cell distribution in the pipe cross section for the three different meshes employed. From left to right: mesh 1, mesh 2, mesh 3, mesh 4.

\section{NUMERICAL METHOD}

When the entire pipe length was simulated the velocity and volume fraction were imposed as boundary conditions at the inlet. It was assumed that the gas enters the pipe through the upper half cross section and the liquid through the bottom half cross section. The inlet velocities where set by using the experimental flow rates of gas and liquid (which were divided by the product of the density and half the pipe cross sectional area). A symmetry condition was imposed at the symmetry plane, and the no-slip boundary condition was applied at the pipe walls. At the outlet, the flow was assumed to be fully developed, and the outflow boundary condition was imposed. As initial condition the volume fraction in the domain was set such that the gas completely fills the upper half of the pipe and the liquid the lower half. The initial values of the velocities and turbulence quantities of the gas and liquid phase were set equal to the values imposed at the pipe inlet for the respective phase.

Periodic boundary conditions were adopted when only a part of the pipe was simulated. Here the pressure drop and hold-up were imposed, thus leaving the gas and liquid flow rates as output quantities resulting from the simulations. It is not 
possible, in fact, to specify the flow rates for multiphase flow, when using periodic boundary conditions in Fluent 17.1. Similarly to the entire pipe simulations a symmetry condition was enforced at the symmetry plane. As initial condition a uniform gas and liquid velocity was set according to the experimental values.

A finite volume method is used to discretize the governing equations. The momentum equation (2) and the turbulence model equations (10) (11) were discretized in space with a second order upwind scheme for the advection terms.

Two different schemes have been used to discretize the volume fraction transport equation (7). In the initial simulations the High Resolution Interface Capturing (HRIC) scheme [23] was adopted, which is a modified version of the Muzaferija et al. scheme [25], but in the final simulations the Geo-reconstruct scheme [23] was employed .

The PRESTO scheme was used to interpolate the pressure at the faces, while the gradients were evaluated with the least squares cell-based method which is a default option in Fluent [23]. The surface tension was modelled with the continuum surface force model proposed by Brackbill et al. [26].

Time dependent calculations were performed in all the simulations, because of the unsteady nature of the waves which appear at the gas-liquid interface. The entire pipe simulations were conducted until the values of the hold-up, pressure gradient and wall friction were almost constant and the remaining temporal oscillation could be attributed to the waves developed at the interface. At every time step the holdup and pressure gradient were evaluated at a fixed location, which was chosen in 
accordance with the location of the measurement devices in the experiments. The time needed to reach the pseudo steady state condition varies between the simulations and it generally lies between 30 and 100 seconds of physical time. After reaching the pseudo steady state the simulation was run for some additional time (more than 20 seconds) and the results were averaged over that time. In the case of periodic boundary conditions, the simulations were run until stable waves characteristics were reached, which generally took between 50 and 140 seconds of physical time.

To solve the governing equations (1) (2) (7) (10) (11) for a time step, a first order implicit discretization scheme was used, for which the HRIC scheme is adopted. Instead an explicit method was used when adopting the Geo-reconstruct scheme, since Fluent 17.1 does not allow to use the implicit time scheme with the Georeconstruct. A fixed time step between 0.005 and 0.02 second has been imposed according to the mesh refinement, and the maximum number of iterations for each time step was set to 35 . The pressure-based solver was selected, and the SIMPLE algorithm was employed to couple the pressure field with the velocity field.

\section{RESULTS}

\section{Full pipe length simulations}

Simulations for two phase air-water flow in a pipe were initially performed and the results were compared both with the experimental results by Espedal [4], and with the CFD results by Lo and Tomasello [15], who used the commercial CFD package Star-CD. The simulations were conducted with mesh 1 (see Table 2), which is the 
same mesh as used by Lo and Tomasello. The equation for the volume fraction was discretized with the HRIC scheme.

The simulated experimental conditions from the experiment by Espedal [4] are given in Table 3 .The pipe is $18 \mathrm{~m}$ long, $0.5^{\circ}$ degree downward inclined and has a diameter of $0.06 \mathrm{~m}$. The density and the dynamic viscosity of the air and the water were set $\rho_{G}=1.225\left[\mathrm{~kg} / \mathrm{m}^{3}\right], \mu_{G}=1.823 \times 10^{-5}[\mathrm{~Pa} \mathrm{~s}]$ and $\rho_{L}=1000\left[\mathrm{~kg} / \mathrm{m}^{3}\right], \mu_{L}=9.91 \times 10^{-4}[\mathrm{~Pa} \mathrm{~s}]$, respectively. The surface tension was set to $0.0715[\mathrm{~N} / \mathrm{m}]$.

Table 3: Experimental flow parameters by Espedal [4] for a $0.06 \mathrm{~m}$ diameter pipe with $0.5^{\circ}$ downward inclination. $U_{S L}$ and $U_{S G}$ are the liquid and gas superficial velocity, respectively; " $a$ " is the wave amplitude. According to definition by Espedal, small amplitude waves have a wave height below $2 \mathrm{~mm}$, the wave length is usually 2$6 \mathrm{~cm}$ and the power spectrum has maximum one peak. Small amplitude 2D waves have characteristics similar to small amplitude waves but they have two peaks in the power spectrum.

\begin{tabular}{cccll}
\hline Case & $\begin{array}{c}U_{S L} \\
(\mathrm{~m} / \mathrm{s})\end{array}$ & $\begin{array}{c}U_{S G} \\
(\mathrm{~m} / \mathrm{s})\end{array}$ & \multicolumn{1}{c}{ Waves } & $a / D(-)$ \\
\hline 1 & 0.12 & 3 & Small Amplitude & 0.00623 \\
2 & 0.18 & 3 & Small Amplitude & 0.00649 \\
3 & 0.26 & 3 & Small Amplitude & 0.0107 \\
4 & 0.32 & 3 & Small Amplitude 2D & 0.011 \\
\hline
\end{tabular}

The $k$ - $\omega$ SST turbulence model was adopted, either without damping or with damping using $B=250$. As shown in Figure 2 , the present results without damping are in agreement with the CFD results of Lo and Tomasello, which thus confirms that the mesh and settings are similar. Also in agreement with the results by Lo and Tomasello is that the pressure gradient in our results without damping is largely over-predicted and the hold-up is under-predicted. When the turbulence at the interface is damped (with $B=250$ in the expression of Egorov), better predictions are obtained especially for the pressure drops which are now close to the experimental values (within $\pm 10 \%$ ). The hold-up is still under- predicted (by close to $10 \%$ ). When 
damping is applied, the prediction of the pressure drop has improved considerably more than the prediction of the hold-up; this is the case both in the present calculations and in those of Lo and Tomasello.

Simulations were also conducted to replicate the air-water pipe flow experiments by Birvalski [17]. Mesh 1 was employed, together with the HRIC discretization scheme. The simulated experimental conditions are given in table 4. The fully horizontal pipe was $10.3 \mathrm{~m}$ long and has a diameter of $0.05 \mathrm{~m}$. The properties of the air and the water used in the calculations were $\rho_{G}=1.225\left[\mathrm{~kg} / \mathrm{m}^{3}\right], \mu_{G}=1.823 \times 10^{-5}[\mathrm{~Pa} \mathrm{~s}]$ and $\rho_{L}=1000\left[\mathrm{~kg} / \mathrm{m}^{3}\right], \mu_{L}=9.91 \times 10^{-4}[\mathrm{~Pa} \mathrm{~s}]$, respectively. The surface tension was set to $0.0715[\mathrm{~N} / \mathrm{m}]$. The $k-\omega$ SST turbulence model was tested both without and with damping. The tuneable parameter $B$ was set to 250 for all the cases with damping. But also simulations with a variation of the value of $B$ were carried out.

Table 4: Experimental flow parameters by Birvalski [17]. $U_{S L}$ and $U_{S G}$ are the liquid and gas superficial velocity, respectively; LA= Large Amplitude.

\begin{tabular}{ccccccccc}
\hline Case & $\begin{array}{c}U_{S L} \\
(\mathrm{~m} / \mathrm{s})\end{array}$ & $\begin{array}{c}U_{S G} \\
(\mathrm{~m} / \mathrm{s})\end{array}$ & Waves & $\begin{array}{c}f_{p} \\
(\mathrm{~Hz})\end{array}$ & $\begin{array}{c}\lambda / D \\
(-)\end{array}$ & $\begin{array}{c}a / D \\
(-)\end{array}$ & $\begin{array}{c}\text { Steepness } \\
(-)\end{array}$ & $\begin{array}{c}c \\
(\mathrm{~m} / \mathrm{s})\end{array}$ \\
\hline 141 & 0.0509 & 3.58 & 2D LA & 5.44 & 0.91 & 0.0186 & 0.13 & 0.49 \\
142 & 0.0509 & 4.12 & 2D LA & 4.69 & 0.96 & 0.0203 & 0.13 & 0.45 \\
143 & 0.0509 & 4.66 & 2D LA & - & 0.96 & 0.0219 & 0.14 & - \\
144 & 0.0509 & 5.02 & 2D LA & 4.35 & 1.09 & 0.0214 & 0.12 & 0.47 \\
\hline
\end{tabular}

To check the influence of tuneable parameter $B$ on the results, various values were tried $(25,250,2500)$ for the case with the lower superficial gas velocity (case 141). Figure 3 compares the predictions for the pressure drop and hold-up with the experiments. The simulation results for the Birvalski experiment are quite similar to what was found for the Espedal experiment (Figure 2); this means that a considerable improvement is obtained for the pressure drop when the damping is 
applied, while the hold-up is under-predicted both with and without damping. The influence of the $B$ value is large for low values of $B(B<250)$. Nonetheless, the results obtained with $B=250$ and with $B=2500$ are rather comparable, and only a difference by $\pm 2 \%$ is found in the prediction of the pressure drop and of the hold-up. This suggests that the sensitivity to $B$ decreases for large values of $B$; more testing, however, is needed for a claim that the results become truly independent in the limit of $B$ tending to infinite.

We also carried out a mesh convergence study for the case with the lower superficial gas velocity of Birvalski (case 141). Simulations were conducted with the $k-\omega$ SST turbulence model both without and with damping at the gas-liquid interface. The predictions for the pressure drop and liquid hold-up, as given in Table 5, converge smoothly for an increasing number of grid cells in the $k-\omega$ SST model without damping. However, for the $k-\omega$ SST model with damping the value of the pressure drop decreases between mesh 1 and mesh 2 and then increases slightly between mesh 2 and mesh 3, suggesting that the results are not fully mesh-independent. This must be due to the built-in mesh dependence of the damping source in the Egorov, as was already noticed by Lo and Tomasello [15]. In fact, the size of the cells near the interface has an influence on the source term in equation (12), both explicitly, with the term $\Delta n$, and implicitly with the term $A$. An in-depth investigation seems to be needed to replace the Egorov damping function by a mesh-independent variant, but this is not part of the present study. The results obtained with mesh 2 are used for further comparison. 
Table 5: Mesh convergence study for case 141 of Birvalski [17]. The experimental values for hold-up and pressure gradient are $0.236(-)$ and $11.8(\mathrm{~Pa} / \mathrm{m})$ respectively. The HRIC scheme was employed.

\begin{tabular}{|c|c|c|c|c|}
\cline { 2 - 5 } \multicolumn{1}{c|}{} & \multicolumn{2}{c|}{$k-\omega$ SST } & \multicolumn{2}{c|}{$k-\omega$ SST damping } \\
\cline { 2 - 5 } \multicolumn{1}{c|}{} & $\begin{array}{c}\mathrm{dP} / \mathrm{dx} \\
(\mathrm{Pa} / \mathrm{m})\end{array}$ & Hold-up (-) & $\begin{array}{c}\mathrm{dP} / \mathrm{dx} \\
(\mathrm{Pa} / \mathrm{m})\end{array}$ & Hold-up (-) \\
\hline Mesh 1 & 24.6 & 0.0980 & 12.1 & 0.178 \\
\hline Mesh 2 & 22.2 & 0.1057 & 8.97 & 0.173 \\
\hline Mesh 3 & 21.3 & 0.1060 & 9.05 & 0.170 \\
\hline
\end{tabular}

Additional simulations were conducted with mesh 2 for the experimental cases by Birvalski, but now by using the Geo-reconstruct discretization scheme for the volume fraction instead of the HRIC scheme. The turbulence was incorporated through the damped $k-\omega$ SST model and by using $B=250$. The hold-up predictions are in better agreement with the experimental results with the Geo-reconstruct scheme (Figure 4) than with the HRIC scheme (Figure 3). Considerably better results are also obtained with the Geo-reconstruct scheme for the axial velocity; see Figure 5. Table 6 shows a comparison between the performance of the $k-\omega$ SST, either without damping using the HRIC scheme, with damping using the HRIC scheme, and with damping using the Geo-reconstruct scheme (for case 141 and mesh 2). The predictions are considerably better when employing damping using the Geo-reconstruct scheme. The considerable difference in results when employing the Geo-reconstruct instead of the HRIC can be due to the resolution (sharpness) of the interface. When employing the HRIC the interface is less sharp than when employing the Geo-reconstruct scheme, thus affecting the magnitude of the interface area density $A$ (gradient of volume fraction) in the damping source term, equation (12). Figure 6 shows the volume fraction and the $\omega$ contours plots for case 141 of Birvalski. It can be seen that 
$\omega$ at the interface is considerably higher when employing the Geo-reconstruct scheme, specifically at locations where the interface is sharper.

Table 6: Discrepancy between predictions and experiments of the pressure drop and hold-up, for case 141 of Birvalski [17]. Mesh 2 was used in all the simulations with $B=250$.

\begin{tabular}{lcccc}
\hline & $\begin{array}{c}\text { Pressure } \\
\text { drop } \\
(\mathrm{Pa} / \mathrm{m})\end{array}$ & $\begin{array}{c}\text { Error } \\
\text { (CFD- } \\
\text { Exp)/Exp }\end{array}$ & $\begin{array}{c}\text { Hold-up } \\
(-)\end{array}$ & $\begin{array}{c}\text { Error } \\
\text { (CFD- } \\
\text { Exp)/Exp }\end{array}$ \\
\hline Experiments Birvalski & 11.8 & - & 0.236 & - \\
\hline$k-\omega$ SST no damping & 22.2 & $+88 \%$ & 0.106 & $-55 \%$ \\
\hline$k-\omega$ SST damp HRIC & 9.0 & $-24 \%$ & 0.173 & $-27 \%$ \\
\hline$k-\omega$ SST damp Geo & 12.8 & $+9 \%$ & 0.259 & $+10 \%$ \\
reconstruct & & & & \\
\hline
\end{tabular}

\section{Simulations with periodic boundary condition: waves analysis}

In stratified gas-liquid flows, the interfacial flow behaviour is of large importance in defining the momentum exchange between the phases. The spatio-temporal characteristics of interfacial waves determine the distribution and intensity of both viscous and turbulent stresses in the interfacial region, see for instance [27]. See also section 6 for a discussion.

Due to their inherent nature, RANS models are not particularly suitable to reproduce such interfacial waves and the associated turbulence structures [28]. Other methods such as LES or DNS are preferable [29-32], but these are much more computationally expensive. Therefore, the authors think that it remains very meaningful to assess the actual capabilities of RANS in capturing interfacial waves.

Simulations were conducted with mesh 4 (see Table 2) in a $1 \mathrm{~m}$ long pipe using periodic boundary conditions (pressure drop). By simulating only a part of the experimental pipe it was possible to reduce the cell size without increasing the total 
number of cells (as compared to the full pipe length simulation), thus improving the spatial discretization and maintaining the same computational effort.

The properties of the air and the water used in the calculations were $\rho_{G}=1.225$ $\left[\mathrm{kg} / \mathrm{m}^{3}\right], \quad \mu_{G}=1.7894 \times 10^{-5}[\mathrm{~Pa} \mathrm{~s}]$ and $\rho_{L}=998.2\left[\mathrm{~kg} / \mathrm{m}^{3}\right], \mu_{L}=1.003 \times 10^{-3}[\mathrm{~Pa} \mathrm{~s}]$, respectively. The surface tension was set to $0.0715[\mathrm{~N} / \mathrm{m}]$ and only the Georeconstruct scheme was used for the volume fraction equation. The simulated experimental conditions from Ayati et al. $[20,21]$ are given in Table 7 . Case 1 is in the region of wave amplitude growth (the amplitude increases with increasing superficial gas velocity), whereas case 2 is in the region of wave amplitude stagnation (the amplitude remains constant for increasing superficial gas velocity), see [33]. These two cases have been appositely chosen to assess the capability of the CFD approach in reproducing the difference in the wave characteristics for the two regions.

The pressure drop and liquid height were used as input to the calculation. The $k-\omega$ SST turbulence model was tested both without and with damping. Starting from a flat interface, a stable single wave appeared in the simulations after a certain number of time steps corresponding to 50 to 140 seconds of physical time.

Table 7: Experimental flow parameters by Ayati et al. [20,21]. $U_{S L}$ and $U_{S G}$ are the liquid and gas superficial velocity, respectively. $d P / d x$ is the measured pressure drop and $h_{l}$ is the averaged measured liquid level.

\begin{tabular}{cccccc}
\hline Case & $\begin{array}{c}U_{S L} \\
(\mathrm{~m} / \mathrm{s})\end{array}$ & $\begin{array}{c}U_{S G} \\
(\mathrm{~m} / \mathrm{s})\end{array}$ & $\begin{array}{c}d P / d x \\
(\mathrm{~Pa} / \mathrm{m})\end{array}$ & $\begin{array}{c}h_{l} \\
(\mathrm{~m})\end{array}$ & Waves \\
\hline 1 & 0.1 & 1.54 & 3.48 & 0.0445 & 2D Small Amplitude \\
2 & 0.1 & 2.29 & 6.62 & 0.0423 & 2D Large Amplitude \\
\hline
\end{tabular}

A comparison between the experimental and simulated results of the superficial velocities and waves characteristics is given in table 8 both for simulation without 
$(B=0)$ and with damping $(B=250)$. There is a substantial improvement in the prediction of the gas superficial velocity when the turbulence is damped at the gasliquid interface while the liquid superficial velocity is unaffected by the damping. Overall the waves at the interface are not correctly simulated and except for the wave frequency in case 1 the discrepancy with experimental results is well above $\pm 20 \%$ for all the compared wave parameters. The damping is seen to affect the wave characteristics. For case 1 the wave length and wave amplitude decrease when damping is applied while the opposite is true for case 2 . The wave frequency is unaffected by the damping in case 1 and increases in the presence of damping for case 2. A clear influence of the damping on the wave characteristics cannot be established from the present results; in fact, the results remain unsatisfactory either without or with damping.

\section{Velocity profiles}

The profiles for the velocity and for the kinetic energy are shown in Figure 7 and Figure 8 for case 1, and in Figure 9 and Figure 10 for case 2 of Ayati et al. [20,21], together with the additional simulations results. The additional simulations were conducted with the $k-\omega$ SST in single phase flow. The liquid and gas phases were simulated separately and the interface was treated as a moving wall with an imposed velocity that is equal to the experimental value. For the liquid phase the moving wall was always smooth while for the gas phase the wall was either smooth or a surface roughness was imposed (roughness height $0.012 \mathrm{~m}$ for case 1 and $0.018 \mathrm{~m}$ for case 2). In a qualitative sense, the simulation of the velocity profile for the smooth moving wall is closer to the experimental results than the simulation with the rough moving wall. However, the superficial gas velocities are over-predicted by about $45 \%$ when 
using a smooth wall, while the discrepancy is less than $4 \%$ for a rough wall. In both the single-phase flow configuration with a rough wall and in the multiphase configuration, the gas velocity profile is skewed towards the top of the pipe with the maximum located above the experimental maximum. This was also observed for the simulated cases of Birvalski, as shown in Figure 5. According to Birvalski [17] the asymmetry in the gas velocity profile can be interpreted as a consequence of secondary flow due to turbulence anisotropy caused by the non-symmetric flow geometry of the gas phase and by the waves acting as a wall roughness. This is further confirmed by the fact that in both the pipe flow experiments of Birvalski and of Ayati et al. the gas velocity profiles are much more symmetrical than found in the channel flow experiments by Akai et al. [11] and by Fabre et al. [34]. The experimental liquid velocity profiles show the characteristic S-shape with negative gradient close to the interface induced by the presence of waves as described by Birvalski [17]. Neither the multiphase simulations nor the single- phase ones are able to correctly represent the liquid velocity profiles. It is thus surprising that the superficial liquid velocities are predicted within $\pm 6 \%$ by the single-phase simulation with smooth moving wall. 
Table 8: Comparison between experimental results by Ayati et al. [20,21] and simulations, for the superficial gas and liquid velocities, and for the wave parameters. $\lambda$ is the wave length, $a$ is the wave amplitude, $c$ is the wave celerity and the wave steepness is defined as $2 \pi a / \lambda$. The percentage difference between the experimental and the simulated values is reported in bold.

\begin{tabular}{|c|c|c|c|c|c|c|c|c|}
\hline & Case & $\begin{array}{c}U_{S L} \\
(\mathrm{~m} / \mathrm{s})\end{array}$ & $\begin{array}{c}U_{S G} \\
(\mathrm{~m} / \mathrm{s})\end{array}$ & $\begin{array}{c}f_{p} \\
(\mathrm{~Hz})\end{array}$ & $\begin{array}{r}\lambda / D \\
(-)\end{array}$ & $\begin{array}{c}a / D \\
(-)\end{array}$ & $\begin{array}{c}\text { Steepness } \\
(-)\end{array}$ & $\begin{array}{c}c \\
(\mathrm{~m} / \mathrm{s})\end{array}$ \\
\hline Exp. & 1 & 0.1 & 1.54 & 2.96 & 1.79 & 0.012 & 0.042 & 0.53 \\
\hline \multirow[t]{2}{*}{$B=0$} & 1 & 0.09 & 1.18 & 2.7 & 1.23 & 0.045 & 0.23 & 0.33 \\
\hline & & $-10 \%$ & $-23 \%$ & $-9 \%$ & $-32 \%$ & $+300 \%$ & $+380 \%$ & $-38 \%$ \\
\hline \multirow[t]{2}{*}{$B=250$} & 1 & 0.09 & 1.60 & 2.73 & 1.13 & 0.027 & 0.15 & 0.3 \\
\hline & & $-10 \%$ & $+4 \%$ & $-8 \%$ & $-37 \%$ & $+130 \%$ & $+260 \%$ & $-43 \%$ \\
\hline Exp. & 2 & 0.1 & 2.29 & 2.45 & 2.41 & 0.035 & 0.088 & 0.59 \\
\hline \multirow{2}{*}{$B=0$} & 2 & 0.12 & 1.82 & 1.63 & 1.22 & 0.052 & 0.27 & 0.2 \\
\hline & & $+20 \%$ & $-20 \%$ & $-33 \%$ & $-49 \%$ & $+48 \%$ & $+210 \%$ & $-66 \%$ \\
\hline \multirow[t]{2}{*}{$B=250$} & 2 & 0.12 & 2.37 & 1.94 & 1.39 & 0.055 & 0.25 & 0.27 \\
\hline & & $+20 \%$ & $+4 \%$ & $-21 \%$ & $-42 \%$ & $+57 \%$ & $+180 \%$ & $-54 \%$ \\
\hline
\end{tabular}

\section{TURBULENCE DAMPING WITH RANS SIMULATIONS}

The two-way interaction between a turbulent gas flow and propagating interfacial waves is very complex as it may encompass numerous non-linear mechanisms. Momentum exchange between phases results in wave growth and acceleration of the liquid layer through the action of normal and tangential stresses, respectively [35]. Initial stages of wave generation and growth is relatively well understood and may be modelled by means of linear hydrodynamic instability approaches, e.g. the viscous Kelvin-Helmholtz or Orr-Sommerfeld theory [36].

As the waves grow and become steeper - either as they develop downstream in a pipe or channel, or as a result of high gas velocities - non-linear mechanisms such as flow separation and wave breaking may take place, significantly altering the momentum fluxes across the interface. Relatively recent numerical and experimental 
studies have demonstrated that gas flow separation, or more specifically shear-layer separation, may occur when the gas phase flows above waves with steepness greater than $0.1[37,38]$. This process diminishes the contact surface between the phases and results in vorticity shedding above the waves. Although these studies were motivated by air-sea interactions and as such are focused on flow in large channels, valuable insight regarding small-scale interfacial dynamics in gas-liquid pipe flow may be extracted from them. The PIV studies of Ayati et al. [21,39] and of Vollestad et al. [40] demonstrated that interfacial dynamics in gas-liquid pipe flow have numerous features in common with open wind-wave channels. One of such features is shear-layer separation above relatively steep waves.

This process is believed to reduce the momentum transfer from the faster flowing gas phase to the liquid phase underneath it. It also imposes changes to the velocity profile of the gas flow. Note that flow separation and wave-shed vorticity may also occur intermittently above waves with relatively low steepness. This has been observed by all studies mentioned in this section.

Based on these experimental observations and the fact that RANS simulations seem to improve with the application of Egorov's turbulence damping function, it is reasonable to make a connection between these two concepts. Our conjecture is that turbulence damping indirectly captures the reduction of momentum transfer across the interface which is caused by flow separation and/or wave-shed vorticity. At this stage, no attempts are made to draw any quantitative relationship between these two concepts. Instead, this discussion is merely meant as a motivation for future studies to search for a connection between them. A physical parameterization 
of turbulence damping based on the above mentioned experimental findings could potentially solve its dependence on tuning and mesh size.

\section{CONCLUSIONS}

CFD simulations were conducted with the commercial code Fluent 17.1 and the $k-\omega$ SST turbulent model for stratified air-water flow in pipes and the results were compared with the experimental results of Espedal [4], Birvalski [17] and Ayati et al. $[20,21]$. Two types of approaches were used. In the first approach the entire pipe was simulated and the velocities of the two phases were given as input to the calculation. In the second approach only a part of the pipe was simulated with periodic boundary conditions, imposing the pressure drop. This second approach made it computationally possible to employ a refined mesh to reproduce the details of the interfacial waves.

It was confirmed that to improve either the prediction of the pressure drop, when superficial velocities are used as input to the calculation, or the prediction of the gas flow rates, when the pressure drop is used as input, it is necessary to modify the turbulence model. The damping source term proposed by Egorov [16] was employed, showing a considerable improvement of the predicted values of the pressure gradient or gas flow rate. It was found that the Geo-reconstruct scheme gives better results than the HRIC scheme, both for the pressure gradient and for the hold-up. A possible explanation of this difference is that the sharpness of the interface influence the damping source term through the interface area density. When turbulence damping is applied and the entire pipe is simulated, the pressure 
drop and hold-up are predicted within $10 \%$ of the experimental values by Espedal and Birvalski.

A value of the damping coefficient equal to 250 was found to be sufficient for all the simulations conducted and increasing the coefficient value above this number was not found to affect the results considerably. A slight dependence of the damping source term on the mesh size has been found: a $\pm 2 \%$ difference in the prediction of the pressure drop and of the hold-up and was found. This seems to be reasonable. However, a follow-up study is required to investigate whether the applied meshdependent damping function at the interface can be replaced by one that does not contain the mesh size and which is sufficiently generic (i.e. no case-by-case tuning is needed).

For the simulations employing periodic boundary conditions (with imposed pressure drop) the prediction of the wave characteristics (wavelength, amplitude, frequency, steepness, and celerity) was overall unsatisfactory when compared with the experimental values obtained by Ayati et al., both without and with the application of turbulence damping. Also, the simulation results for the gas and liquid velocity profiles were in significant disagreement with the experimental results. The calculated gas velocity profiles had maxima closer to the upper pipe wall than found in the experimental velocity profiles. However, when damping was applied, the gas superficial velocities were within $4 \%$ of the experimental values while the liquid superficial velocities were either under-predicted by $10 \%$ or over-predicted by $20 \%$. Surprisingly, when the liquid phase was simulated independently as a single-phase flow with a smooth moving upper wall, the superficial liquid velocity was within $\pm 6 \%$ 
of the experimental values. Nevertheless, for the gas phase, it was necessary to impose a surface roughness to the moving wall in order to obtain only a small discrepancy in the superficial gas velocity of $4 \%$. Both the liquid and gas velocity profiles were not in agreement with the experimental PIV measurements when the single-phase simulations were conducted, while treating the interface as a moving wall.

The results obtained in the present study demonstrate that performing CFD simulations with RANS, in which only one set of equations is solved for both phases, is not a reliable method for gas-liquid stratified flow, in particular not if the interfacial wave characteristics are of interest. If RANS with VOF is employed it helps to enable the damping source term proposed by Egorov with a damping constant $B$ equal or greater than 250 . Good results were obtained when simulating the two phases separately as single-phase flow streams, with a smooth moving wall for the liquid phase and a rough wall for the gas phase, serving as the interface. Nonetheless, the velocity profiles obtained with the single-phase simulation were not in agreement with the PIV measurements. Care is needed when using such an approach to simulate gas-liquid stratified flow, since a good agreement for the bulk quantities will not necessarily imply a good agreement for the velocity profiles. 

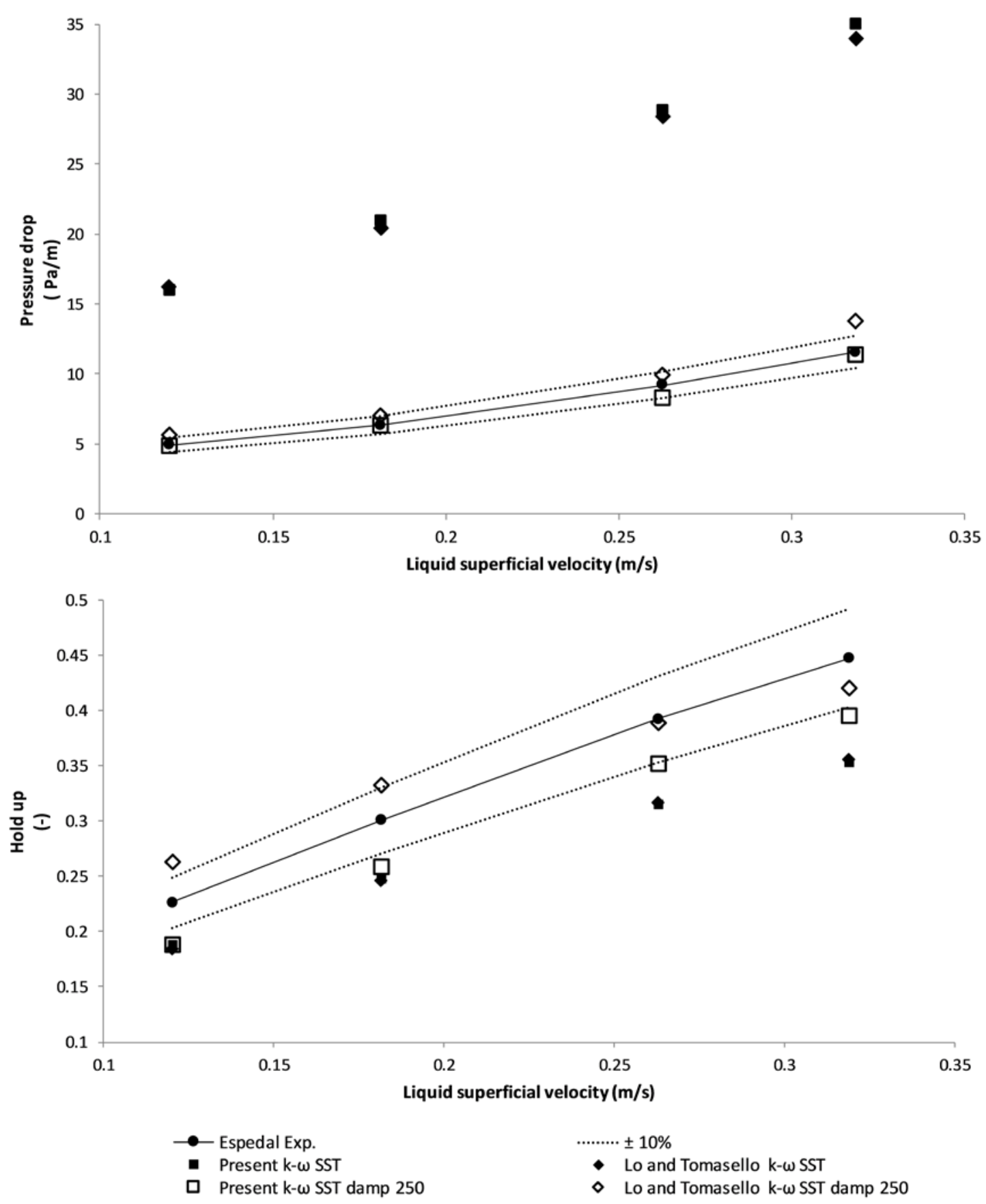

Figure 2: Comparison between the experimental results by Espedal [4], the numerical results by Lo and Tomasello [15] and the present simulations. A damping factor value of $B=250$ and the HRIC scheme have been used in both the present simulations and in the simulations of Lo and Tomasello. Mesh 1 was applied. 

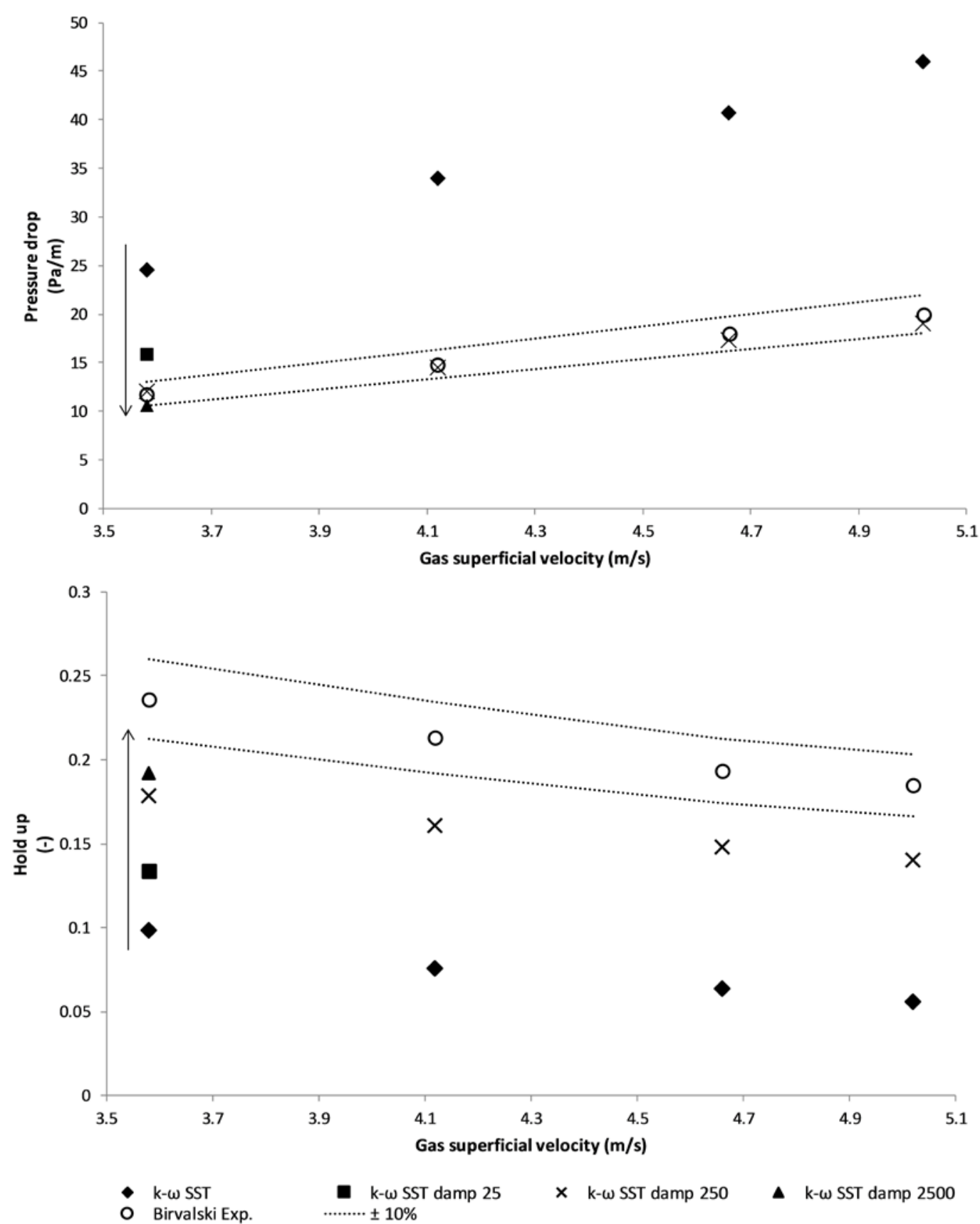

Figure 3: Comparison between the experimental results by Birvalski [17] and the present simulations for different values of the $B$ damping factor $(0,25,250,2500)$, volume fraction discretization scheme HRIC, and using mesh 1. 

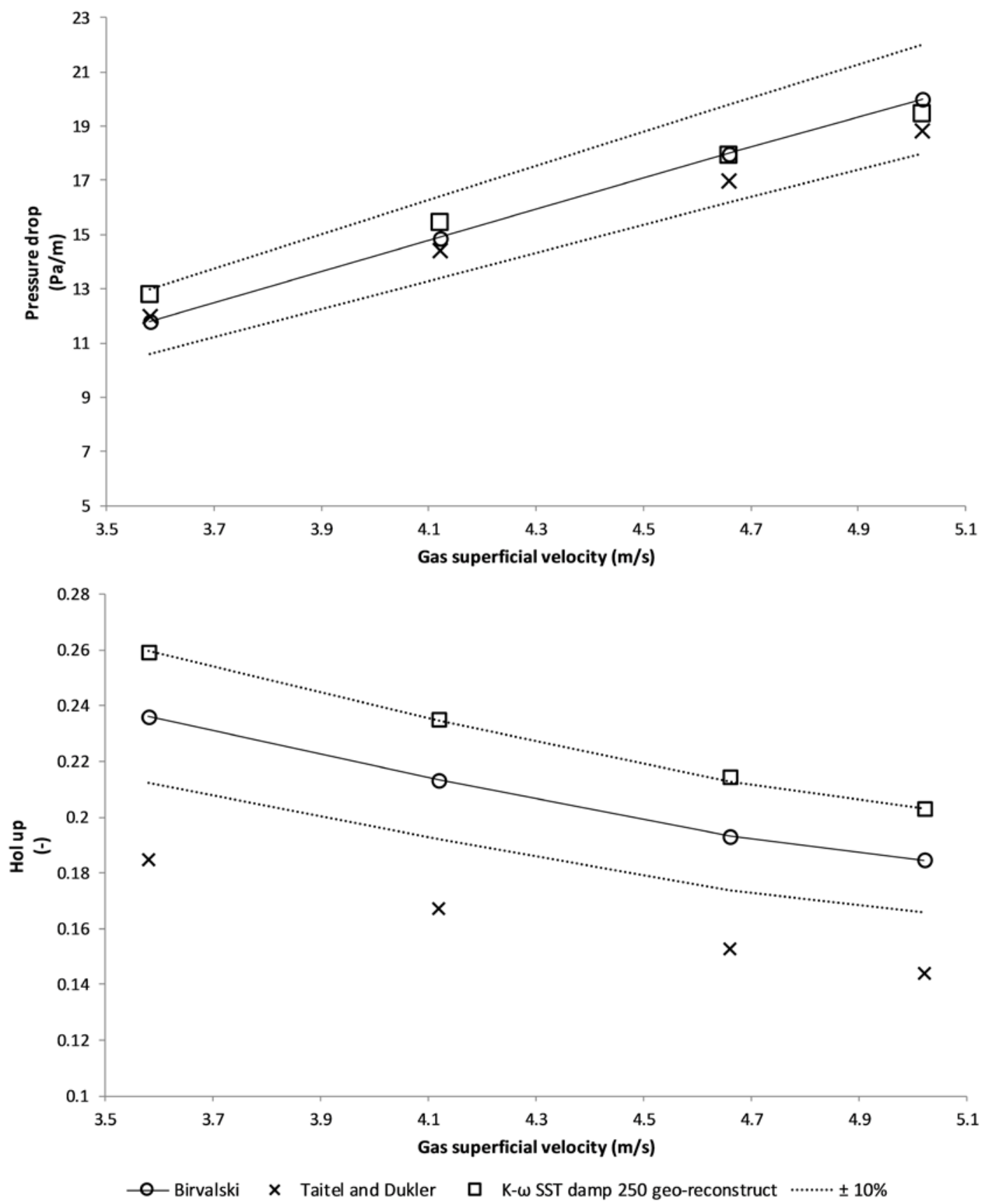

Figure 4: Comparison between the experimental results by Birvalski [17], the present simulations and the Taitel and Dukler model with $f_{\text {int }} / f_{\text {gas }}=3$ [3]. The damping factor $B$ is equal to 250 , volume fraction discretization scheme Geo-Reconstruct, and using mesh 2. 

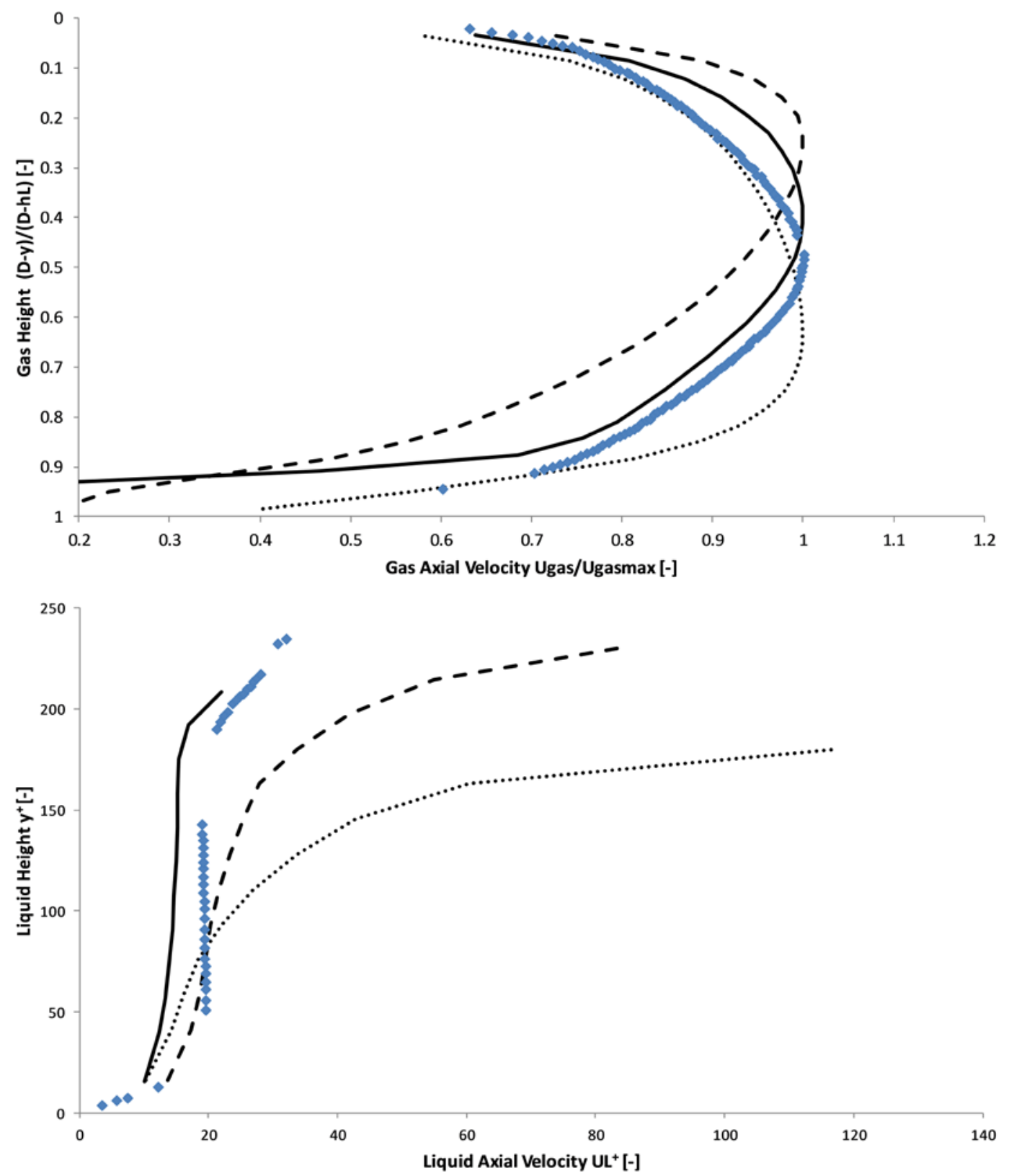

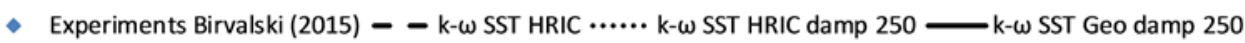

Figure 5: Gas (top) and liquid (bottom) velocity profiles for case 141 of Birvalski [17]. Comparison between the experimental results and the present simulations, for the $k-\omega$ SST HRIC without damping, the $k-\omega$ SST with damping $B=250$ and HRIC scheme, the $k-\omega$ SST with damping $B=250$ and Geo-reconstruct scheme, using mesh 2. 
contour-1

Volume fraction (phase-1)
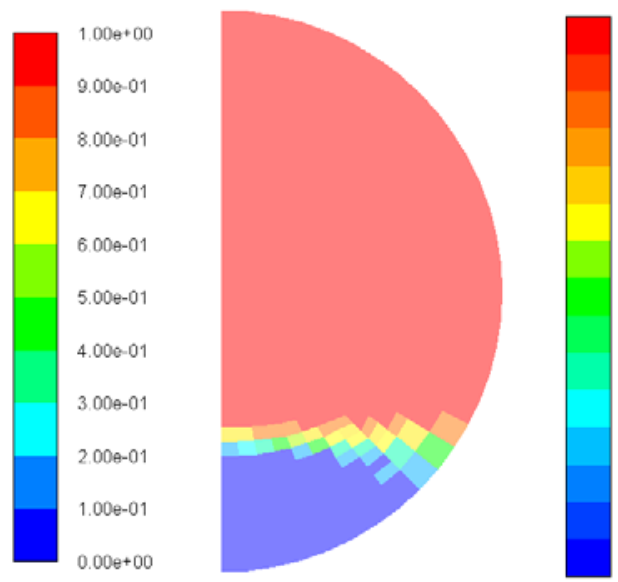

$9.83 e+03$

$9.18 \mathrm{e}+03$

$8.53 e+03$

$7.87 \mathrm{e}+03$

$7.22 e+03$

$6577^{+}+03$

$5910+03$

$5.26 \mathrm{e}+03$

$4.600+03$

$3.950+03$

$3.300+03$

$264 \mathrm{e}+03$

$1.99 e+03$

$1.34 \mathrm{e}+03$

$6.81 e+02$

$[1 / 5]$

$2.76 e+01$

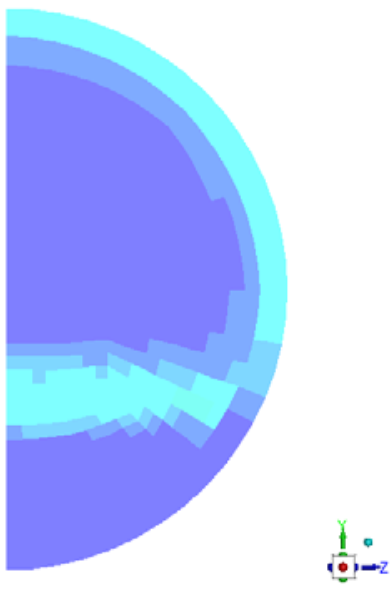

contour-1

contour-1
Volume fraction (phase-1)

soecific Dissipation Rate (Omega) (mixture)
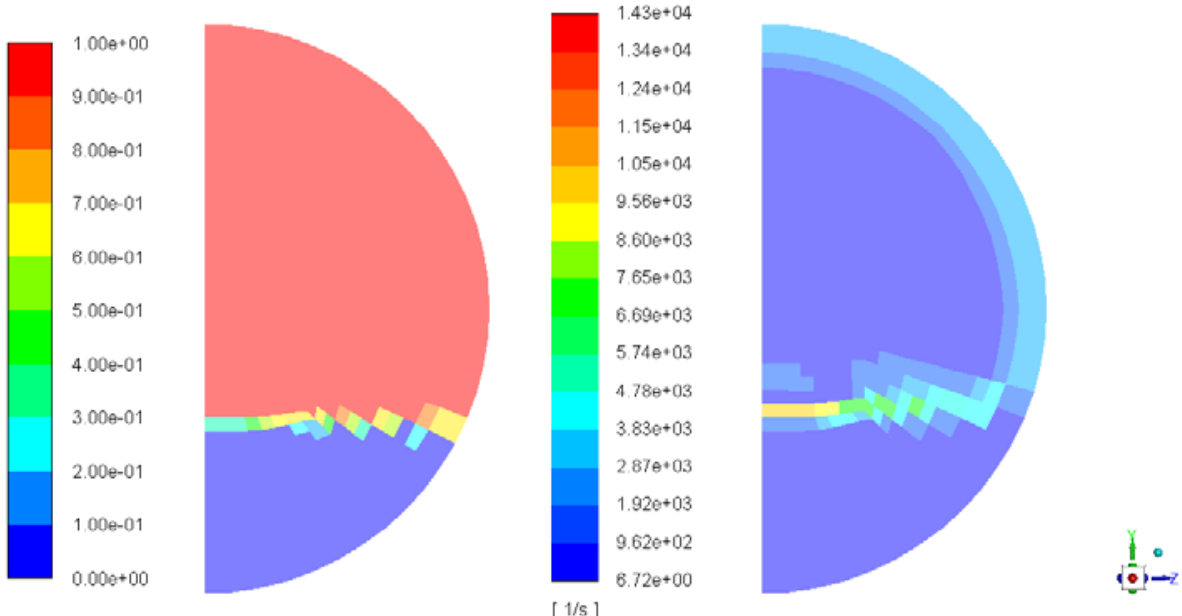

Figure 6: Contours of the volume fraction (left) and the specific dissipation rate $\omega$ (right), for the case 141 of Birvalski employing mesh 2 and $B=250$. Top figures HRIC scheme, bottom figures Geo-reconstruct scheme. 

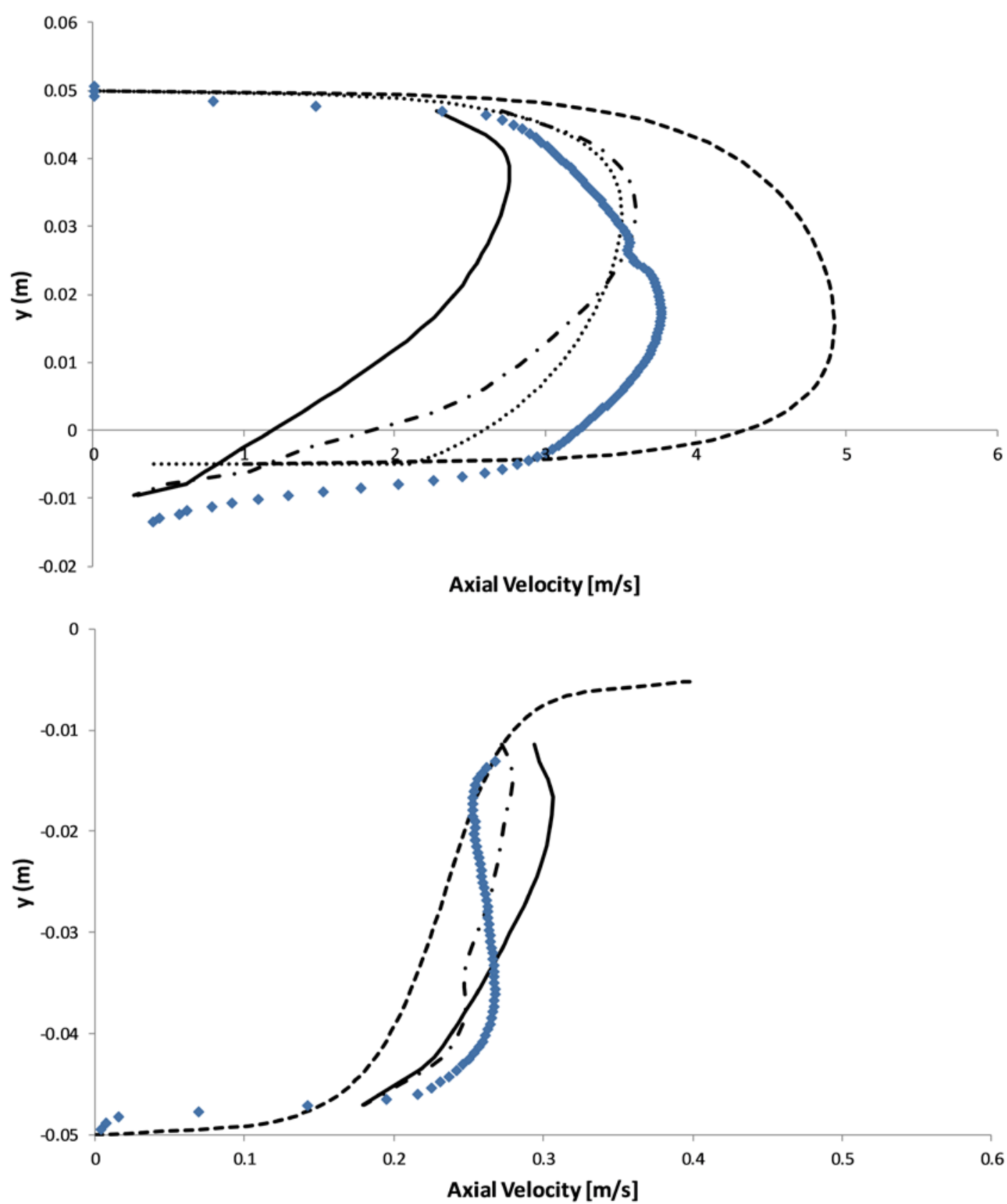

- Exp. — B $=0$ Trough - - B=250 Trough --- Single phase smooth $\cdots \cdots .$. Single phase rough

Figure 7: Gas (top) and liquid (bottom) velocity profiles for case 1 of Ayati et al. Comparison between the experimental results and the present simulations, for the $k-\omega$ SST without damping, the $k-\omega$ SST with damping $B=250$ and $k-\omega$ SST in single phase flow where the interface is simulated by a moving wall. Geo-reconstruct scheme with mesh 4. 

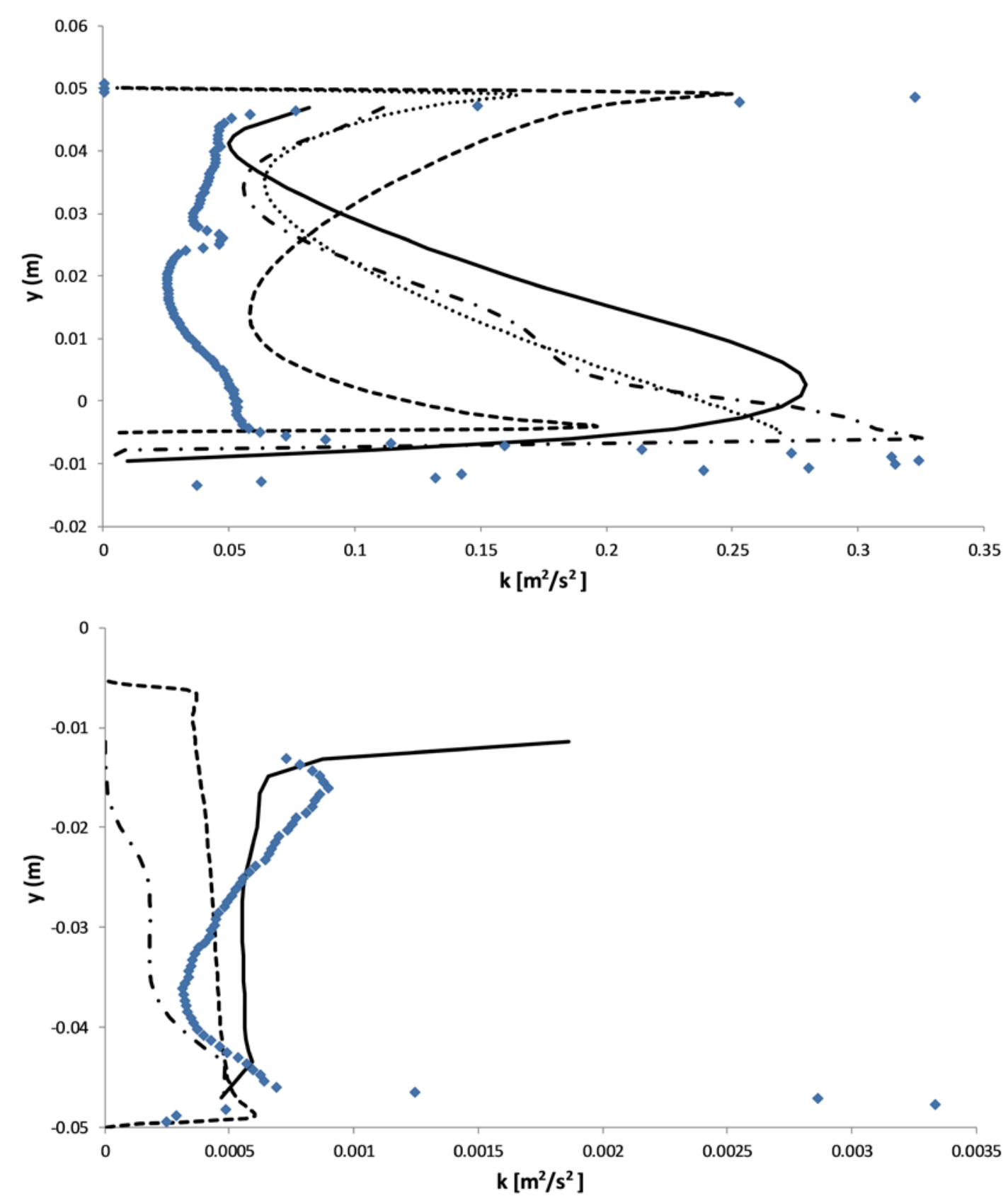

- Exp. $-B=0$ Trough - - $B=250$ Trough --- Single phase smooth $\cdots \cdots \cdot$... Single phase rough

Figure 8: Gas (top) and liquid (bottom) turbulent kinetic energy profiles for case 1 of Ayati et al.. Comparison between the experimental results and the present simulations, for the $k-\omega$ SST without damping, the $k-\omega$ SST with damping $B=250$ and $k-\omega$ SST in single phase flow where the interface is simulated by a moving wall. Georeconstruct scheme with mesh 4. 

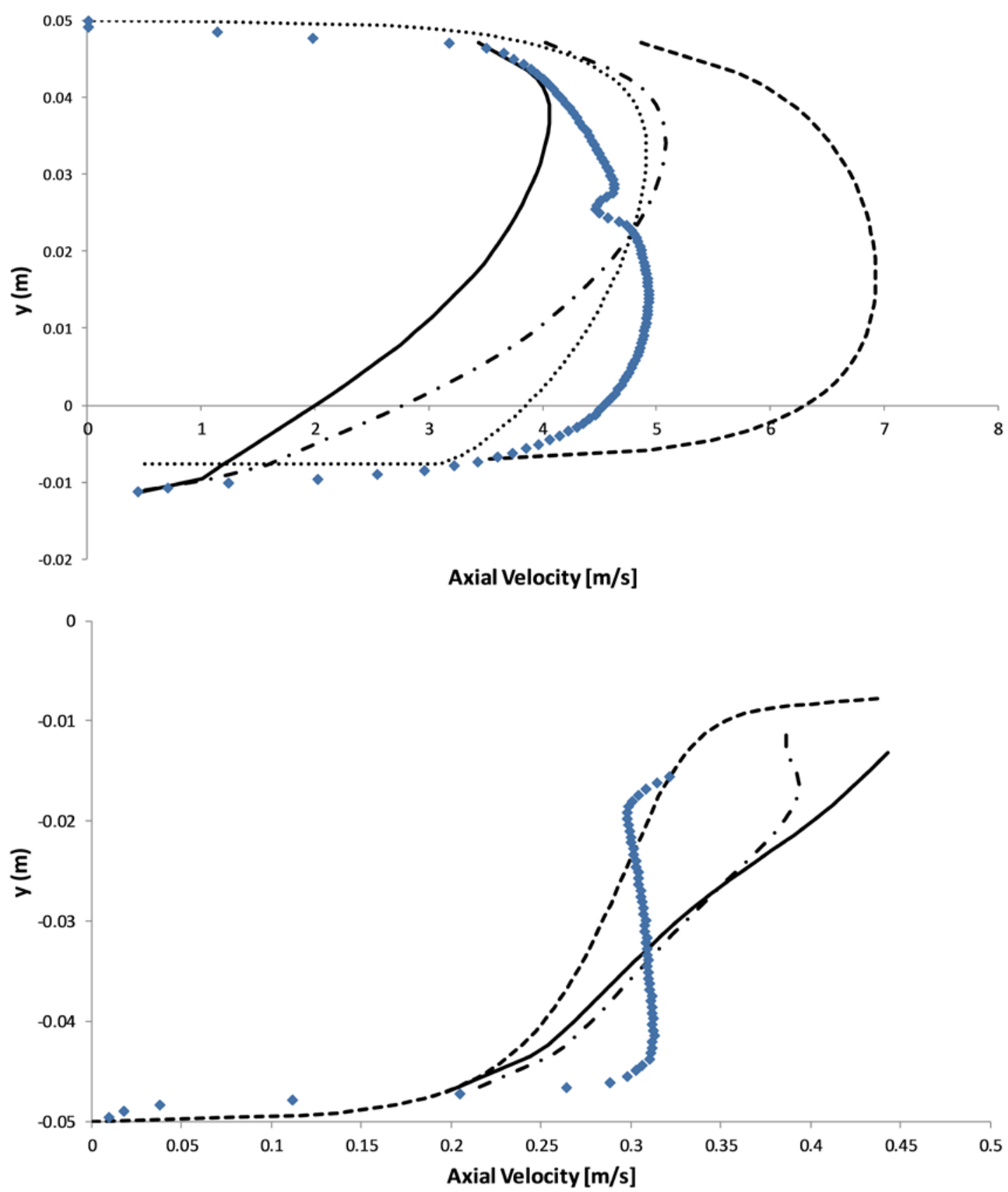

- Exp. — B=0 Trough - - B $=250$ Trough --- Single phase smooth $\cdots \cdots$... Single phase rough

Figure 9: Gas (top) and liquid (bottom) velocity profiles for case 2 of Ayati et al. Comparison between the experimental results and the present simulations, for the $k-\omega$ SST without damping, the $k-\omega$ SST with damping $B=250$ and $k-\omega$ SST in single phase flow where the interface is simulated by a moving wall. Geo-reconstruct scheme with mesh 4. 

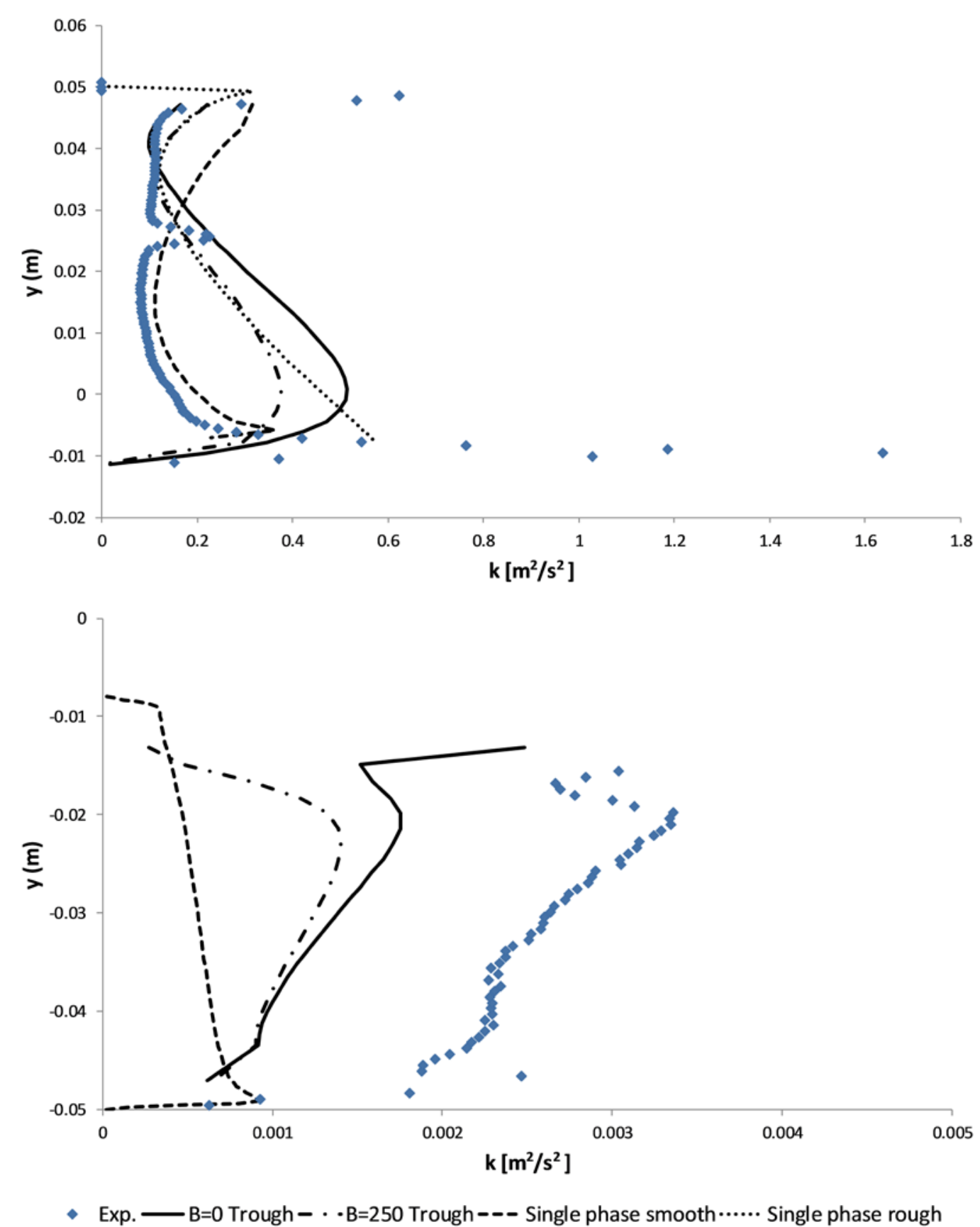

Figure 10: Gas (top) and liquid (bottom) turbulent kinetic energy profiles for case 2 of Ayati et al. Comparison between the experimental results and the present simulations, for the $k$ - $\omega$ SST without damping, the $k-\omega$ SST with damping $B=250$ and $k-\omega$ SST in single phase flow where the interface is simulated by a moving wall. Georeconstruct scheme with mesh 4. 


\section{ACKNOWLEDGMENT}

This work would not have been possible without the assistance of Dr.ir. M. Pourquie and Dr.ir. M. Birvalski.

\section{FUNDING}

The support of TUV-NEL and the Energy Technology Partnership (ETP) in financing the underway PhD study conduct by the first author is gratefully acknowledged.

\section{NOMENCLATURE}

\begin{tabular}{|c|c|}
\hline$A$ & Interface area density \\
\hline$a$ & Wave amplitude, $\mathrm{m}$ \\
\hline$B$ & Turbulence damping tuning parameter \\
\hline$c$ & Wave celerity, $\mathrm{m} / \mathrm{s}$ \\
\hline$D$ & Pipe diameter, $\mathrm{m}$ \\
\hline$d P / d x$ & Axial pressure drops, $\mathrm{Pa} / \mathrm{m}$ \\
\hline$f_{p}$ & Wave peak frequency, $\mathrm{Hz}$ \\
\hline$\vec{g}$ & Gravitational acceleration vector, $\mathrm{m} / \mathrm{s}^{2}$ \\
\hline$h_{L}$ & Liquid height, $\mathrm{m}$ \\
\hline I & Unit tensor \\
\hline$k$ & Turbulence kinetic energy, $\mathrm{m}^{2} / \mathrm{s}^{2}$ \\
\hline$L A$ & Large Amplitude \\
\hline$P$ & Pressure, $\mathrm{Pa}$ \\
\hline
\end{tabular}


Strain rate magnitude, $1 / \mathrm{s}$

$S_{\omega} \quad$ Specific dissipation rate source term, $\mathrm{kg} / \mathrm{m}^{3} \mathrm{~s}^{2}$

$t$

Time, $\mathrm{s}$

$U L^{+} \quad$ Dimensionless liquid velocity

$U_{S L} \quad$ Superficial liquid velocity, $\mathrm{m} / \mathrm{s}$

$U_{S G} \quad$ Superficial gas velocity, $\mathrm{m} / \mathrm{s}$

$\vec{v} \quad$ Velocity vector, $\mathrm{m} / \mathrm{s}$

$\vec{v}^{T} \quad$ Transpose of the velocity vector, $\mathrm{m} / \mathrm{s}$

$\mathrm{y}^{+} \quad$ Dimensionless wall distance

$\alpha_{1} \quad$ Volume fraction of primary phase

$\alpha_{2} \quad$ Volume fraction of secondary phase

$\beta \quad$ Turbulence model constant

$\Delta \mathrm{n} \quad$ Cell height normal to the interface, $\mathrm{m}$

$\lambda \quad$ Wave length, $m$

$\mu \quad$ Dynamic viscosity, Pa s

$\mu_{\mathrm{t}} \quad$ Turbulent viscosity, Pa s

$\rho \quad$ Density, $\mathrm{kg} / \mathrm{m}^{3}$

$\vec{\tau} \quad$ Molecular stress tensor, $\mathrm{Pa}$

$\vec{\tau}_{t} \quad$ Turbulent stress tensor, $\mathrm{Pa}$

$\omega \quad$ Specific dissipation rate, $1 / \mathrm{s}$ 


\section{REFERENCES}

[1] Lockhart, R., and Martinelli, R., 1949, "Proposed Correlation of Data for Isothermal Two-Phase, Two-Component Flow in Pipes," Chem. Eng. Prog., 45(1), pp. 39-48.

[2] Chisholm, D., 1967, "A Theoretical Basis for the Lockhart-Martinelli Correlation for Two-Phase Flow," Int. J. Heat Mass Transf., 10(12), pp. 1767-1778.

[3] Taitel, Y., and Dukler, A., 1976, "A Model for Predicting Flow Regime Transitions in Horizontal and near Horizontal Gas-Liquid Flow," AIChE J., 22(1), pp. 47-55.

[4] Espedal, M., 1998, "An Experimental Investigation of Stratified Two-Phase Pipe Flow at Small Inclinations," Ph.D. thesis, Norwegian University of Science and Technology.

[5] Biberg, D., 2007, "A Mathematical Model for Two-Phase Stratified Turbulent Duct Flow," Multiph. Sci. Technol., 19(1), pp. 1-48.

[6] Grolman, E., and Fortuin, J., 1997, "Gas-Liquid Flow in Slightly Inclined Pipes," Chem. Eng. Sci., 52(24), pp. 4461-4471.

[7] Birvalski, M., and Henkes, R., 2012, "Experiments and Modelling of Multiple Holdup States for Gas/Liquid Flow in a Pipeline," Proceedings of the 8th North American Conference on Multiphase Technology, Banff.

[8] Birvalski, M., Koren, G., and Henkes, R., 2014, "Experiments and Modelling of Liquid Accumulation in the Low Elbow of a Gas/Liquid Pipeline," Proceedings of the 9th North American Conference on Multiphase Technology, Banff.

[9] Prosperetti, A., and Tryggvason, G., 2007, Computational Methods for Multiphase Flow, Cambridge University Press, Cambridge.

[10] Holmås, K., Nossen, J., and Mortensen, D., 2005, "Simulation of Wavy Stratified Two-Phase Flow Using Computational Fluid Dynamics (CFD)," Proceedings of the 12th International Conference on Multiphase Production Technology, Barcelona.

[11] Akai, M., Inoue, A., Aoki, S., and Endo, K., 1980, "A Co-Current Stratified AirMercury Flow with Wavy Interface," Int. J. Multiph. Flow, 6(3), pp. 173-190.

[12] Lorencez, C., Nasr-Esfahany, M., and Kawaji, M., 1997, "Liquid Turbulence Structure at a Sheared and Wavy Gas-Liquid Interface," Int. J. Multiph. Flow, 23(2), pp. 205-226.

[13] Terzuoli, F., Galassi, M., and Mazzini, D., 2008, "CFD Code Validation against Stratified Air-Water Flow Experimental Data," Sci. Technol. Nucl. Install., 2008, pp. 1-7.

[14] Fabre, J., Suzanne, C., and Masbernat, L., 1987, "Experimental Data Set No. 7: Stratified Flow, Part I: Local Structure," Multiph. Sci. Technol., 3(1-4), pp. 285301.

[15] Lo, S., and Tomasello, A., 2010, "Recent Progress in CFD Modelling of Multiphase Flow in Horizontal and Near-Horizontal Pipes," Proceedings of the 7th North American Conference on Multiphase Technology, Banff.

[16] Egorov, Y., 2004, Contact Condensation in Stratified Steam-Water Flow, EVOLECORA D 07.

[17] Birvalski, M., 2015, "Experiments in Stratified Gas-Liquid Pipe Flow," Ph.D. thesis, Delft Univeristy of Technology. 
[18] Birvalski, M., Tummers, M. J., Delfos, R., and Henkes, R. A. W. M., 2014, "PIV Measurements of Waves and Turbulence in Stratified Horizontal Two-Phase Pipe Flow," Int. J. Multiph. Flow, 62, pp. 161-173.

[19] Birvalski, M., Tummers, M. J., and Henkes, R. A. W. M., 2016, "Measurements of Gravity and Gravity-Capillary Waves in Horizontal Gas-Liquid Pipe Flow Using PIV in Both Phases," Int. J. Multiph. Flow, 87, pp. 102-113.

[20] Ayati, A. A., Kolaas, J., Jensen, A., and Johnson, G. W., 2015, "Combined Simultaneous Two-Phase PIV and Interface Elevation Measurements in Stratified Gas/Liquid Pipe Flow," Int. J. Multiph. Flow, 74, pp. 45-58.

[21] Ayati, A. A., Kolaas, J., Jensen, A., and Johnson, G. W., 2016, "The Effect of Interfacial Waves on the Turbulence Structure of Stratified Air/Water Pipe Flow," Int. J. Multiph. Flow, 78, pp. 104-116.

[22] Menter, F. R., 1994, "Two-Equation Eddy-Viscosity Turbulence Models for Engineering Applications," AIAA J., 32(8), pp. 1598-1605.

[23] ANSYS Inc., 2016, ANSYS FLUENT 17.1 User's Guide, Canonsburg, Pennsylvania, United States of America.

[24] Wilcox, D. C., 2006, Turbulence Modeling for CFD, DCW Industries Inc., La Canada, CA.

[25] Muzaferija, S., Peric, M., Sames, P., and Schellin, T., 1998, "A Two-Fluid NavierStokes Solver to Simulate Water Entry," Proceedings of the 22nd Symposium on Naval Hydrodynamics, Washington, DC, pp. 277-289.

[26] Brackbill, J., Kothe, D., and Zemach, C., 1992, "A Continuum Method for Modeling Surface Tension," J. Comput. Phys., 100(2), pp. 335-354.

[27] André, M. A., and Bardet, P. M., 2017, "Viscous Stress Distribution over a Wavy Gas-liquid Interface," Int. J. Multiph. Flow, 88, pp. 1-10.

[28] Bestion, D., Coste, P., Niceno, C., Mimouni, S., Lakehal, D., and Bartosiewicz, Y., 2011, "Two-Phase CFD: The Various Approaches and Their Applicability to Each Flow Regime," Multiph. Sci. Technol., 23, pp. 101-128.

[29] Lakehal, D., Fulgosi, M., Yadigaroglu, G., and Banerjee, S., 2003, "Direct Numerical Simulation of Turbulent Heat Transfer Across a Mobile, Sheared Gas-Liquid Interface," J. Heat Transfer, 125(6), pp. 1129-1139.

[30] Lakehal, D., and Liovic, P., 2011, "Turbulence Structure and Interaction with Steep Breaking Waves," J. Fluid Mech., 674, pp. 522-577.

[31] Lombardi, P., De Angelis, V., and Banerjee, S., 1996, "Direct Numerical Simulation of Near-Interface Turbulence in Coupled Gas-Liquid Flow," Phys. Fluids, 8(6), pp. 1643-1665.

[32] Christensen, E. D., 2006, "Large Eddy Simulation of Spilling and Plunging Breakers," Coast. Eng., 53(5-6), pp. 463-485.

[33] Ayati, A. A., and Carneiro, J. N. E., 2018, "Statistical Characterization of Interfacial Waves in Turbulent Stratified Gas-Liquid Pipe Flows," Int. J. Multiph. Flow.

[34] Fabre, J., Masbernat, L., and Suzanne, C., 1987, "Stratified Flow, Part I: Local Structure," Multiph. Sci. Technol., 3(7), pp. 285-301.

[35] Grare, L., Peirson, W. L., Branger, H., Walker, J. W., Giovanangeli, J. P., and Makin, V., 2013, "Growth and Dissipation of Wind-Forced, Deep-Water Waves," J. Fluid Mech., 722, pp. 5-50.

[36] Barmak, I., Gelfgat, A., Vitoshkin, H., Ullmann, A., and Brauner, N., 2016, 
"Stability of Stratified Two-Phase Flows in Horizontal Channels," Phys. Fluids, 28(4), pp. 44-101.

[37] Yang, D., and Shen, L., 2010, "Direct-Simulation-Based Study of Turbulent Flow over Various Waving Boundaries," J. Fluid Mech., 650, pp. 131-180.

[38] Buckley, M. P., and Veron, F., 2016, "Structure of the Airflow above Surface Waves," J. Phys. Oceanogr., 46(5), pp. 1377-1397.

[39] Ayati, A. A., Vollestad, P., and Jensen, A., 2018, "Detailed Measurements of Interfacial Dynamics in Air-Water Pipe Flow," Procedia IUTAM, 26, pp. 59-69.

[40] Vollestad, P., Ayati, A. A., Angheluta, L., LaCasce, J. H., and Jensen, A., 2018, "Experimental Investigation of Airflow above Waves in a Horizontal Pipe," Under Rev. Int. J. Multiph. Flow. 


\section{Figure Captions List}

Fig. 1 Cell distribution in the pipe cross section for the three different meshes employed. From left to right: mesh 1, mesh 2, mesh 3, mesh 4.

Fig. 2 Comparison between the experimental results by Espedal [4], the numerical results by Lo and Tomasello [15] and the present simulations. A damping factor value of $B=250$ and the HRIC scheme have been used in both the present simulations and in the simulations of Lo and Tomasello. Mesh 1 was applied.

Fig. 3 Comparison between the experimental results by Birvalski [17] and the present simulations for different values of the $B$ damping factor $(0,25,250,2500)$, volume fraction discretization scheme HRIC, and using mesh 1.

Fig. 4 Comparison between the experimental results by Birvalski [17], the present simulations and the Taitel and Dukler model with $f_{\text {int }} / f_{\text {gas }}=3$ [3]. The damping factor $B$ is equal to 250, volume fraction discretization scheme GeoReconstruct, and using mesh 2.

Fig. 5 Gas (top) and liquid (bottom) velocity profiles for case 141 of Birvalski [17]. Comparison between the experimental results and the present simulations, for the $k-\omega$ SST HRIC without damping, the $k-\omega$ SST with damping $B=250$ and HRIC scheme, the $k$ - $\omega$ SST with damping $B=250$ and Geo-reconstruct scheme, using mesh 2.

Fig. 6 Contours of the volume fraction (left) and the specific dissipation rate $\omega$ (right), for the case 141 of Birvalski employing mesh 2 and $B=250$. Top figures HRIC scheme, bottom figures Geo-reconstruct scheme.

Fig. 7 Gas (top) and liquid (bottom) velocity profiles for case 1 of Ayati et al. Comparison between the experimental results and the present simulations, for the $k-\omega$ SST without damping, the $k-\omega$ SST with damping $B=250$ and $k-\omega$ SST in single phase flow where the interface is simulated by a moving wall. Georeconstruct scheme with mesh 4.

Fig. 8 Gas (top) and liquid (bottom) turbulent kinetic energy profiles for case 1 of Ayati et al.. Comparison between the experimental results and the present simulations, for the $k-\omega$ SST without damping, the $k-\omega$ SST with damping $B=250$ and $k-\omega$ SST in single phase flow where the interface is simulated by a moving wall. Geo-reconstruct scheme with mesh 4. 
Fig. 9 Gas (top) and liquid (bottom) velocity profiles for case 2 of Ayati et al. Comparison between the experimental results and the present simulations, for the $k-\omega$ SST without damping, the $k-\omega$ SST with damping $B=250$ and $k-\omega$ SST in single phase flow where the interface is simulated by a moving wall. Georeconstruct scheme with mesh 4.

Fig. 10 Gas (top) and liquid (bottom) turbulent kinetic energy profiles for case 2 of Ayati et al. Comparison between the experimental results and the present simulations, for the $k-\omega$ SST without damping, the $k-\omega$ SST with damping $B=250$ and $k-\omega$ SST in single phase flow where the interface is simulated by a moving wall. Geo-reconstruct scheme with mesh 4. 


\section{Table Caption List}

Table $1 \quad$ Constants in the $k$ - $\omega$ SST turbulence model [23]

Table 2 Cell distribution of the three different meshes employed

Table 3 Experimental flow parameters by Espedal [4] for a $0.06 \mathrm{~m}$ diameter pipe with $0.5^{\circ}$ downward inclination. $U_{S L}$ and $U_{S G}$ are the liquid and gas superficial velocity, respectively; " $a$ " is the wave amplitude. According to definition by Espedal, small amplitude waves have a wave height below $2 \mathrm{~mm}$, the wave length is usually $2-6 \mathrm{~cm}$ and the power spectrum has maximum one peak. Small amplitude 2D waves have characteristics similar to small amplitude waves but they have two peaks in the power spectrum.

Table 4 Experimental flow parameters by Birvalski [17]. $U_{S L}$ and $U_{S G}$ are the liquid and gas superficial velocity, respectively; $L A=$ Large Amplitude.

Table 5 Mesh convergence study for case 141 of Birvalski [17]. The experimental values for hold-up and pressure gradient are $0.236(-)$ and $11.8(\mathrm{~Pa} / \mathrm{m})$ respectively. The HRIC scheme was employed.

Table 6 Discrepancy between predictions and experiments of the pressure drop and hold-up, for case 141 of Birvalski [17]. Mesh 2 was used in all the simulations with $B=250$.

Table 7 Experimental flow parameters by Ayati et al. [20,21]. $U_{S L}$ and $U_{S G}$ are the liquid and gas superficial velocity, respectively. $d P / d x$ is the measured pressure drop and $h_{l}$ is the averaged measured liquid level.

Table 8 Comparison between experimental results by Ayati et al. [20,21] and simulations, for the superficial gas and liquid velocities, and for the wave parameters. $\lambda$ is the wave length, $a$ is the wave amplitude, $c$ is the wave celerity and the wave steepness is defined as $2 \pi \mathrm{a} / \lambda$. The percentage difference between the experimental and the simulated values is reported in bold. 
Journal of Fluids Engineering 